\title{
Behavioral programming with the APPLE II microcomputer
}

\author{
GLENN C. THOMPSON \\ Duke University, Durham, North Carolina 27706
}

\begin{abstract}
A system is described that allows behavioral programming with an APPLE II microcomputer. Input and output interfaces were designed with relays so that maximum flexibility could be obtained with a variety of behavioral chambers and boxes. The system is inexpensive and easily constructed and requires no special knowledge of computers to program or maintain.
\end{abstract}

Despite recent advances in the design of low-cost multipurpose computers, many laboratories continue to choose relay and solid state logic modules for controlling behavioral experiments. This may be because relay logic is cheaper (often it is already on hand); it is simple to interface to behavioral apparatus; it is simple to program; no special skills are needed to operate it; and it has proved dependable over the years. Thus, in spite of their initial low cost, in the long run, computers may be more expensive than relay and solid state logic because of hidden costs such as those associated with the need for specialized maintenance, as well as with the need for hardware and software programming specialists. Without such ancillary services, computers may also be functionally less versatile than relays for the special problems that the behaviorist encounters.

Most behaviorists would probably have little objection in using a computer system that would (1) be initially inexpensive, (2) be easily interfaced to behavioral apparatus, (3) be easily programmed (i.e., with high-level languages such as BASIC or FORTRAN, (4) require little or no maintenance, and (5) be dependable. This paper describes a microcomputer system that appears to meet these criteria.

\section{GENERAL DESCRIPTION OF APPLE II MICROCOMPUTER SYSTEM}

The APPLE II microcomputer is a self-contained unit based on the MOS-6502 microprocessor; it comes with a power supply, random-access memory (RAM; up to $48 \mathrm{~K}$ ), resident integer BASIC on read-only memory (ROM), cassette and video interfaces, and motherboard with room for up to seven expansion

The system described in this paper was supported by NIMH Grant 04849 to I. T. Diamond, NIH Grant 05584 to G.C. Thompson, and NIH Biomedical Research Support Grant to Duke University. I would like to thank Dr. Diamond for his encouragement and support during the automation of his behavioral laboratory. The author's present address is the Department of Otorhinolaryngology and Communicative Sciences, Baylor College of Medicine, Texas Medical Center, Houston, Texas 77030 . cards. Other features of the APPLE II include TV. screen graphics, full color graphics (with color TV), and four analog-to-digital (A/D) converters, two of which are connected to game-control paddles. The APPLE II also comes with an extensive reference manual and a separate BASIC programming manual written at an instructional level for users with no previous programming experience. For the experienced programmer, a powerful system monitor allows interaction at the assembly language level. Although the APPLE II is well supported by hardware accessories such as disk operating systems and serial and parallel output cards, the system described here requires none of these.

To make the APPLE II into a complete behavioral programmer, the items shown in Table 1 are essential.

\section{INPUT INTERFACE}

One of the unique features that sets the APPLE II apart from similar microcomputers is its on-board $A / D$ converter. There are four such devices built into the APPLE II that can be accessed via a game input/output (I/O) connector. In addition, there are three switchclosure inputs available on this connector that make input interfacing extremely simple. For example, if a particular experiment requires only one, two, or three separate response manipulanda, each can be input directly

Table 1

Materials Required for the APPLE II

\begin{tabular}{lr}
\hline & $\begin{array}{c}\text { Approximate } \\
\text { Cost }\end{array}$ \\
\hline APPLE II with 16K RAM & $\$ 1,200$ \\
Small video monitor or TV & 100 \\
RF modulator for use only with TV & 30 \\
Cassette recorder for storing programs and data & 50 \\
Input interface* (5 manipulanda @\$5) & 25 \\
Output interface*: & 24 \\
APPLE prototype card & 20 \\
ICs and sockets & 40 \\
Eight relays & $<\$ 1,500$ \\
Total &
\end{tabular}

*See text for description. 
by providing a switch closure between Pins 2 and 1 $(+5 \mathrm{~V})$ for the first manipulandum, Pins 3 and $1(+5 \mathrm{~V})$ for the second manipulandum, or Pins 4 and $1(+5 \mathrm{~V})$ for the third manipulandum. To determine when a particular response has been made requires one simple line of BASIC:

10 IF PEEK $(-16285)>127$ THEN (some line number): GOTO $10^{1}$

This statement looks at memory location -16285 and determines if the value is greater than 127 . If it is, then the switch has been closed and a response has been made, and control jumps to wherever the program dictates. If the value is less than 127 , the statement after the colon is executed: The computer goes back to the beginning of the line and checks the memory location again to see if the value has changed. Similarly, PEEK $(-16286)$ and PEEK $(-16287)$ provide information about the other two inputs.

Fortunately, three independent inputs can satisfy the requirements of a wide variety of behavioral paradigms. When more are needed, there is another inputting strategy that utilizes the same $\mathrm{I} / \mathrm{O}$ connector.

Our use required eight separate manipulanda, so we constructed an input interface that makes use of one of the two game paddles that connect to the A/D converter via the same game $I / O$ connector. For instance, each game paddle contains a potentiometer that changes resistance from 0 to $150 \mathrm{kohms}$ as the lever is moved; the A/D converter "reads" the resistance and "converts" it to a number between 0 and 255 . Thus, each number represents a unique range of resistances lying between 0 and $150 \mathrm{kohms}$. To input an animal's response to the computer, it is necessary only to substitute a fixed resistor for the potentiometer. This can be accomplished by placing a resistor across the normally open contacts of a switch attached to a manipulandum operated by the animal. Figure 1 shows a simple circuit diagram for inputting responses from several independent manipulanda to the computer.

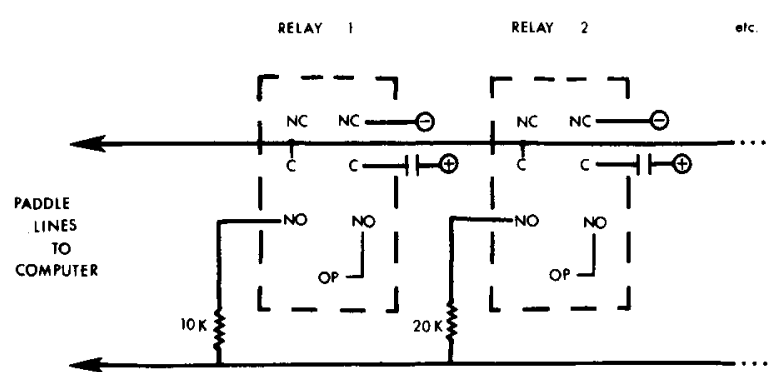

Figure 1. Schematic diagram for inputting responses from multiple manipulanda into the APPLE II. Each manipulandum operates one of these relays, which in turn places a unique resistance across the paddle lines. The capacitor discharges through the relay coil and keeps it closed for a standard minimum duration. The paddle levers can be cut off to allow access to the lines.

\section{SOFTWARE PROGRAMMING OF INPUTS}

Software programming on the APPLE II is accomplished in integer BASIC. Since this very fast version of BASIC is resident in ROM, no RAM memory is used; all 16K of RAM is available for programs and data. The floating-point version of BASIC is APPLEsoft. APPLEsoft contains more powerful commands than does integer BASIC but it runs significantly slower and uses valuable RAM space. Since the extended commands are not needed, APPLEsoft is not recommended for behavioral programming.

In order to program the computer to accept responses from some manipulandum, a special function exists that facilitates input from the game paddles. For example, the BASIC statement "X=PDL(1)" places the value of $X$ equal to a number between 0 and 255 , which corresponds to the resistance the A/D converter reads from Paddle 1. In this manner, up to 255 separate inputs can be identified if each has a unique resistance lying between 0 and $150 \mathrm{kohms}$.

Since relays are generally used to switch the appropriate resistor into the A/D converter line going into the computer, two inherent characteristics of these devices must be dealt with: (1) Since relays take a finite time to close, the resistance between the normally open contacts changes rapidly until they are firmly closed; and (2) relay contacts usually bounce as they close. Both of these characteristics are potentially troublesome for the computer if it is to accurately read the fixed resistor momentarily placed across the relay contacts. To alleviate these problems, the computer must first sense that a response-any response-has been made; then it must be restrained from reading the resistance until the bouncing relay contacts are stable; finally, it must read the resistance before the relay begins to open again.

The software program can delay the computer from reading the resistance until the relay contacts have firmly closed simply by placing the computer into a timing loop. To insure that the relay remains closed long enough for the computer to get an accurate reading (even when the animal's actual response is very brief), a capacitor is placed into the circuit so that it discharges through the relay coil when the relay is energized, thus keeping it on for a time (see Figure 1). The following BASIC statements will input and then identify a particular response:

\section{$10 \mathrm{X}=$ PDL(1): IF $\mathrm{X}<255$ THEN 20: GOTO 10 20 FOR $K=1$ TO 5 : NEXT K: X=PDL(1) 30 RESPONSE $=V(X)$.}

Statement 10 instructs the computer to read Paddle 1. Since a resistance greater than $150 \mathrm{kohms}$ (i.e., open relay contacts) returns " $X=255$," the computer remains in Statement 10 until a response is made; the response then actuates a relay and places a resistance less than 
150 kohms across the A/D converter line. Statement 20 , the debounce statement, instructs the computer to read Paddle 1 only after it has counted from one to five (or any other number that delays sufficiently long). These two lines allow the computer to sense a response and to identify the manipulandum on which it was made. But one more step is helpful-one that identifies the manipulandum in absolute terms. This can be accomplished by one of two methods. The first method uses more memory space than the second, but it executes faster and does not require that the resistors used to identify the manipulanda be of any specific value. To use this method, a variable table " $V$ " is constructed that has a maximum of 256 entries corresponding to all possible outcomes of the value of $X$ (from 0 to 255). Next, each entry is given a value that corresponds to one manipulandum. Thus, $\mathrm{V}(1) \cdot \mathrm{V}(10)$ may all be set to 1 to denote Manipulandum 1; V(11)-(20) may be set to 2 to denote Manipulandum 2; V(21)-(30) may be set to 3 to denote Manipulandum 3, and so on. The end result is that each entry of variable table $V$ has an identifier that specifies a particular manipulandum. Statement 30 instructs the computer to go to that location in the variable table $\mathrm{V}$ specified by $\mathrm{X}$ in Line 20 and to place the value that resides in that location into a new variable called RESPONSE. Of course, the variable table $\mathrm{V}$ has to be set to the proper values somewhere in the program before Statement 30 is executed. These values should be determined empirically and depend upon the exact values of the resistors used to identify each response. Because the look-up table $V$ has an entry for each value of $X$, the exact resistor values selected are not critical. However, the best results with this method have been obtained with resistor values less than 100 kohms.

The second method uses less memory and is simpler and more efficient to program. But it requires that the resistors selected have values that result in the translated numbers returned by the $A / D$ converter being nearly equidistant from each other. If this constraint is met, then Line 30 can be:

\section{RESPONSE $=((X-1) /$ number of increments} in translated numbers per manipulandum) +1 .

For example, if resistors are selected for three manipulanda so that the translated numbers are 5,15 , and 25 , then

\section{RESPONSE $=((X-1) / 10)+1$}

Thus, when the first manipulandum is operated and a 5 is returned by the computer: RESPONSE $=((5-1) / 10)+1$. This results in RESPONSE $=1$, since $4 / 10$ in integer BASIC equals 0. Similarly, when the second manipulandum is operated, which returns a 15 , RESPONSE= $((15-1) / 10)+1=14 / 10+1=2$ in integer arithmetic.
Although computer experts may feel that this particular strategy of inputting responses to the APPLE II is less than ideal, there are two strong points in its favor: (1) For the behaviorist who is most familiar with relay logic, it is a simple thing to do and can be implemented without the aid of a technician; and (2) it works.

\section{OUTPUT INTERFACE}

To control experimental parameters, the APPLE II must be able to turn external devices on and off at specified times. Because of the variety of devices used by different experimenters in the behavioral sciences, rather than build specialized circuits for driving each unique device, we constructed a universal interface. The interface can drive relays that the computer can turn on or off, and its contacts can be used for switching almost any external electrical device. Figure 2 illustrates a relay driver interface built on an APPLE II prototype card using a combination of integrated circuits and transistors, solder, and wire-wrap techniques. Placement and construction are not critical. Once constructed, the card can be plugged into one of the seven expansion slots available in the computer and can control up to eight external relays.

\section{OUTPUT SOFTWARE PROGRAMMING}

Special BASIC commands facilitate control of the relays. POKE $X, Y$ places the value of $Y$ directly into the memory location $X$. Because the manufacturers of the APPLE II have already coded access to the expansion

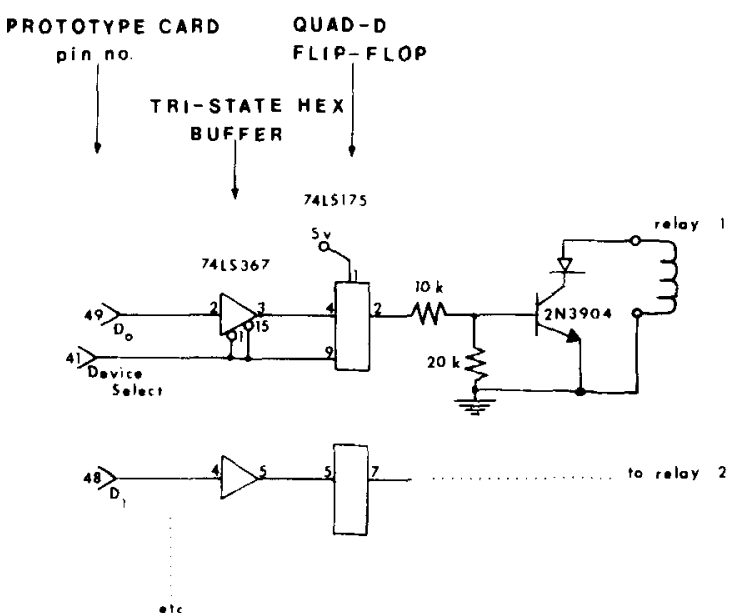

Figure 2. Schematic diagram for driving relays from the APPLE II. Each of eight drivers is identical to the one shown. The common side of the relay coils should be tied to the ground on this card, but the relays themselves should be placed externally. Two .01-microfarad capacitors should be placed on the prototype board according to instructions that come with it. 
slots and to the $\mathrm{I} / \mathrm{O}$ lines in those slots (which are used to enable the relay drivers), it is extremely easy to command the relays. A few examples will illustrate: Slot 5's memory location is -16176 , so if the relaydriver card is plugged into Slot 5, "POKE - 16176,1" will write a 1 to the relay-driver card. This turns on Relay 1; "POKE -16176,2" turns on Relay 2; "POKE $-16176,4$ " turns on Relay 3; and "POKE - 16176,5" turns on both Relays 1 and 3. It should be apparent that the $\mathrm{I} / \mathrm{O}$ lines are binary coded so that any combination of the eight relays (up to 255) can be turned on by placing a single number in the POKE statement. To turn all relays off, " POKE -16176,0" will do. Code can be written to identify the output lines in terms of their function and to make the program more readable. For example:

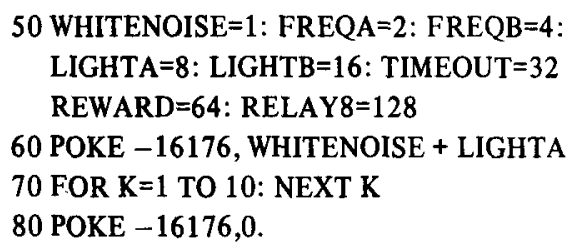

Line 50 identifies the function of each relay, as well as the computer line that will control it. Line 60 turns on two relays, one that controls white noise and one that controls Light A. Line 70 is a timing loop and Line 80 turns off all relays. Although eight output relays can control a wide variety of behavioral paradigms, some experiments may require more. A simple solution is to construct a second relay-driver interface card and plug it into another expansion slot. From BASIC, it takes approximately $30 \mathrm{msec}$ to turn on a relay driven from one slot after turning on a relay driven from a different slot, but for most uses this time delay is not critical.

\section{OTHER SOFTWARE PROGRAMMING CONSIDERATIONS}

An important facet to almost any control program is the ability to time external devices. Timing loops can be programmed in BASIC with "FOR ... NEXT" statements. Within the programmed timing loop, statements can be written that allow the computer to check whether or not a response has been made. Of course, any statements that reside inside the loop increase the time taken to execute the loop. Thus, when writing timing loops, it is imperative to place inside the loop all interruptions that are necessary for the final program and then to calibrate the loop using a timer or oscilloscope. As an example, the following short program turns Relay 1 on and off at programmable intervals so that calibration can be accomplished:

\author{
10 POKE $-16176,1$ \\ 20 FOR L=1 TO $\mathrm{Z}$ : $\mathrm{X}=\mathrm{PDL}(1)$ : IF $\mathrm{X}<255$ THEN 50: \\ NEXT L \\ 30 POKE $-16176,0$ \\ 40 FOR $L=1$ TO $Z$ : $X=P D L(1)$ : IF $X<255$ THEN 50: \\ NEXT L: GOTO 10 \\ 50 PRINT "A RESPONSE INTERRUPTED THE \\ LOOP”: GOTO 10.
}

To calibrate, place a voltage through the normally open contacts of Relay 1 and into an oscilloscope. Then set $Z=100$ and run the program. The relay turns on for whatever duration the loop takes to count to $\mathrm{Z}$, all the while checking to make sure a response has not occurred. It then turns off for the same length of time, and then on again, and so on. By placing a few different values for $Z$, the loop can be calibrated conveniently.

Other programming techniques are straightforward for anyone familiar with BASIC and easy to learn from the manuals provided with the APPLE II for anyone unfamiliar with BASIC.

\section{USING THE APPLE II SYSTEM}

The system described in this paper has been used in our laboratory for more than 18 months and is very reliable. Our only problem was a power supply failure. The power supply was replaced immediately by the local APPLE II dealer.

Because of its modular design and low cost, repair on the APPLE II can realistically be accomplished by replacement. This strategy requires no specialized repairmen and reduces down-time significantly, a real advantage for researchers. Software programming time has been minimal; entirely new programs have been written, debugged, and readied for use between $24-\mathrm{h}$ experimental sessions.

Before the APPLE II, laboratory personnel used relay logic with Mack-panel programming to control all contingencies and to record data from a completely automated, seven-choice, free-field, sound-localization apparatus. The programming racks were cumbersome, expensive, and eventually required almost constant maintenance. The APPLE II alleviated these problems, and our data collection became uneventful and much more reliable. Furthermore, we can now analyze the data immediately and plot it on the TV screen.

\section{NOTE}

1. APPLE II integer BASIC treats a colon as the end of a statement line. Thus, multiple statements can be written on one line with a single line number.

(Received for publication August 20, 1979; revision accepted October $15,1979$. ) 